\title{
IL4-Pseudomonas Exotoxin Fusion Protein MDNA55
}

National Cancer Institute

\section{Source}

National Cancer Institute. IL4-Pseudomonas Exotoxin Fusion Protein MDNA55. NCI

Thesaurus. Code C79843.

A fusion protein consisting of the cytokine interleukin-4 (IL-4) linked to a truncated form of Pseudomonas exotoxin with potential antineoplastic activity. Upon specific, highaffinity binding to IL-4 receptors located on the tumor cell surface., IL4-Pseudomonas exotoxin fusion protein MDNA55 is internalized; the exotoxin moiety then binds to translation elongation factor 2 (EF-2), which may result in ADP ribosylation, deactivation of EF-2, inhibition of protein synthesis, and tumor cell apoptosis. The Pseudomonas exotoxin moiety of this agent has been engineered to reduce non-specific binding to cells expressing its receptor, the multiligand cell surface receptor alpha 2-macroglobulin receptor/low-density lipoprotein receptor-related protein (alpha 2MR/LRP). IL-4R is a type I transmembrane protein that binds IL-4 and IL-13 and may be overexpressed by cancers such as renal cell carcinoma and glioma. 